\title{
Mott transition in the two-dimensional flux phase
}

\author{
Y. Otsuka ${ }^{1}$ and Y. Hatsugai ${ }^{1,2}$ \\ ${ }^{1}$ Department of Applied Physics, University of Tokyo, 7-3-1 Hongo Bunkyo-ku, Tokyo 113-8656, Japan \\ ${ }^{2}$ PRESTO, Japan Science and Technology Corporation, Kawaguchi-shi, Saitama 332-0012, Japan
}

(Received 9 April 2001; revised manuscript received 29 August 2001; published 14 January 2002)

\begin{abstract}
Effects of the electron-electron interaction in the two-dimensional flux phase are investigated. We treat the half-filled Hubbard model with a magnetic flux $\pi$ per plaquette by the quantum Monte Carlo method. When the interaction is small, an antiferromagnetic long-range order does not exist. It suggests that the Mott transition occurs at finite strength of the interaction in the flux phase, which is in contrast to the standard Hubbard model.
\end{abstract}

DOI: 10.1103/PhysRevB.65.073101

PACS number(s): 71.10.Fd, 71.30. $+\mathrm{h}$

\section{INTRODUCTION}

There has been a proposal that an order takes place on a link in several interacting lattice-fermion systems. Especially when the link order has a phase factor, it brings an effective magnetic field. Sometimes the order can be topological where the phase factor itself is not a well defined order parameter but the flux characterizes the phase. One such system is the flux phase which was proposed to describe the ground state properties of several interacting lattice-fermion systems, e.g., the Hubbard model, the $t-J$ model, and their variants. ${ }^{1-7}$ Recently there has been a resurgence of interest in the flux phase and evidences are accumulating for its reality. For example, it was revealed that bond order takes place in the one-dimensional extended Hubbard model at half filling ${ }^{8}$ which is the one-dimensional analog of the flux phase. Further, it has been discussed that a hidden topological order exists in cuprates, which shares some aspects with the flux phase. ${ }^{9}$ In general, however, the flux phase competes with other instabilities, e.g., superconductivity, antiferromagnetism, charge order, and localization due to disorder (see Refs. 10-13 for the effects of disorder on the flux phase). In particular, the flux phase often competes with superconductivity, which is a direct consequence from the SU(2) symmetry at half filling. ${ }^{2}$ We also note that the flux phase can emerge dynamically ${ }^{5,6}$ as well as in a static form. ${ }^{9}$

In this paper, we investigate effects of the interaction in the two-dimensional flux phase. We choose the twodimensional Hubbard model with a magnetic flux $\phi=\pi$ per square plaquette and compare its properties with those of the standard Hubbard model $(\phi=0)$. In the standard Hubbard model at half filling, an infinitesimally small interaction drives the ground state to the Mott insulator, where charge gap opens and an antiferromagnetic long-range order exists. This is a consequence from the nesting properties of the Fermi surface in the language of the SDW mean field theory for the weak coupling region. On the other hand, in the flux phase without the interaction, the density of states disappears linearly at the Fermi energy, which suggests that the structure of the low energy excitations is singular as compared with a simple Fermi liquid and the nesting instability is absent. Therefore one can expect an interaction-driven quantum phase transition from a singular quantum liquid (density of states is linearly vanishing without interaction) to a gapped insulator (Mott insulator).

\section{FLUX PHASE}

The flux phase is given by the ground state of the following simple Hamiltonian:

$$
\mathcal{H}_{F}=\sum_{\langle j, k\rangle, \sigma}\left(c_{j \sigma}^{\dagger} t_{j k} c_{k \sigma}+c_{k \sigma}^{\dagger} t_{k j}^{*} c_{j \sigma}\right),
$$

where $\langle j, k\rangle$ denotes a nearest-neighbor link. The amplitude of $t_{j k}$ is constant but its phase factor $t_{j k} /\left|t_{j k}\right|=e^{i \theta_{j k}}$ satisfies a condition $\phi=\Sigma_{\text {plaquette }} \theta_{j k}$. It leads to a uniform magnetic flux per plaquette. The phase factor $\theta_{j k}$ itself is not fixed but the flux $\phi$ is fixed, which is gauge independent. This Hamiltonian was proposed as an effective model of several correlated electron systems and discussed in many different contexts. ${ }^{1-7,11-16}$ One of the focuses was the stability of the flux state. ${ }^{14-16}$ Following the discussion, the optimum, energy-minimizing, magnetic flux at half filling (this is the simplest case) is considered as $\pi$ per square plaquette. ${ }^{14,15}$ Furthermore, we note that Lieb gave some rigorous results for the stability in a general form. ${ }^{16}$

At half filling, the low-lying excitations of the flux phase is described by massless Dirac fermions. There is a gauge freedom for the phase factor $\theta_{j k}$ but let us fix them by choosing as $t_{j+\hat{x}, j}=(-1)^{j_{y}} t, t_{j+\hat{y}, j}=t$ and otherwise zero where $j=\left(j_{x}, j_{y}\right) \in \mathbf{Z}^{2}, \hat{x}=(1,0), \hat{y}=(0,1)$. The energy bands in this gauge are given by

$$
\begin{aligned}
E(\mathbf{k}) & = \pm 2 t \sqrt{\cos ^{2} k_{x}+\cos ^{2} k_{y}} \\
& \approx \pm 2 t \sqrt{\left(k_{x}-k_{x}^{i}\right)^{2}+\left(k_{y}-k_{y}^{i}\right)^{2}} \quad(i=1,2),
\end{aligned}
$$

where $\left(k_{x}, k_{y}\right) \in[-\pi, \pi) \times[0, \pi), \mathbf{k}^{1}=\left(k_{x}^{1}, k_{y}^{1}\right)=(\pi / 2, \pi / 2)$, and $\mathbf{k}^{2}=\left(k_{x}^{2}, k_{y}^{2}\right)=(-\pi / 2, \pi / 2)$. Therefore the low-lying excitations are described by massless Dirac fermions at these two gap-closing points and the density of states $D(\epsilon)$ near the Fermi energy vanishes linearly, $D(\epsilon) \propto|\epsilon|$. The density of states $D(\epsilon)$ is singular and it leads to the strong suppression of the antiferromagnetic instability as discussed below. Note that the dispersion is gauge dependent but the density of states is gauge independent. We focus on only the gauge independent quantity in this paper. 


\section{MODEL AND METHOD}

We investigate effects of the interaction in the flux phase by the following Hamiltonian:

$$
\begin{aligned}
\mathcal{H}= & \sum_{\langle j, k\rangle, \sigma}\left(c_{j \sigma}^{\dagger} t_{j k} c_{k \sigma}+c_{k \sigma}^{\dagger} t_{k j}^{*} c_{j \sigma}\right) \\
& +U \sum_{i}\left(n_{i \uparrow}-1 / 2\right)\left(n_{i \downarrow}-1 / 2\right),
\end{aligned}
$$

where $\langle j, k\rangle$ denotes a nearest-neighbor link and $U$ a on-site Coulomb repulsion. The geometry is set to be a twodimensional square lattice and a periodic boundary condition is imposed. The grand-canonical ensemble is employed and we put the system half filled by the particle-hole symmetry. The $\left|t_{j k}\right|$ is set to be constant $(=t)$ and, based on the Lieb theorem, ${ }^{16}$ the phase factor $e^{i \theta_{j k}}$ is chosen so that the magnetic flux $\phi$ is $\pi$ per plaquette, i.e., $\pi$-flux phase ( $\phi$ $\left.\equiv \Sigma_{\text {plaquette }} \theta_{j k}=\pi\right)$. We always tries to compare the results of the flux phase $(\phi=\pi)$ with those of the standard Hubbard model $(\phi=0)$. It is to be noted that time-reversal symmetry is not broken in the $\pi$-flux phase of our gauge.

In order to study the system based on a nonperturbative approximation free method, the quantum Monte Carlo (QMC) technique is applied. ${ }^{17,18}$ We use the grand-canonical scheme at finite temperatures. Due to the particle-hole symmetry, the negative-sign problem does not occur. The simulations were performed on a square lattice with a size up to $N=12 \times 12$ at a temperature down to $T=0.05 t$. The Trotter decomposition is performed in the imaginary-time direction and the time slice is $\Delta \tau \simeq 0.10 / t$. We have checked that the systematic errors due to the Trotter decomposition are almost independent of temperatures and does not change the essential features after the extrapolation. We have typically performed 500 Monte Carlo sweeps in order to reach a thermal equilibrium followed by 5000 measurement sweeps. The measurements are divided into 10 blocks and the statistical error is defined by the variance among the blocks.

The Mott insulator is characterized by the following two features. One is a strong suppression of the charge fluctuation and the other is a presence of the strong antiferromagnetic spin correlation. In order to detect signals of the Mott transition, we have calculated the charge compressibility and the magnetic structure factor. The charge compressibility is defined by

$$
\kappa=\frac{1}{N} \frac{\partial N_{e}}{\partial \mu}=\frac{\beta}{N}\left(\left\langle N_{e}^{2}\right\rangle-\left\langle N_{e}\right\rangle^{2}\right),
$$

where $N_{e}$ is the number of electrons and $\beta$ an inverse temperature. The charge compressibility $\kappa$ measures the charge fluctuation directly. If the system has a finite charge gap, $\kappa$ shows a thermally activated behavior and vanishes at zero temperature. The magnetic structure factor is given by

$$
S(\boldsymbol{q})=\frac{1}{N} \sum_{i, j} e^{i \boldsymbol{q} \cdot\left(\boldsymbol{r}_{i}-\boldsymbol{r}_{j}\right)}\left\langle\left(n_{i \uparrow}-n_{i \downarrow}\right)\left(n_{j \uparrow}-n_{j \downarrow}\right)\right\rangle .
$$

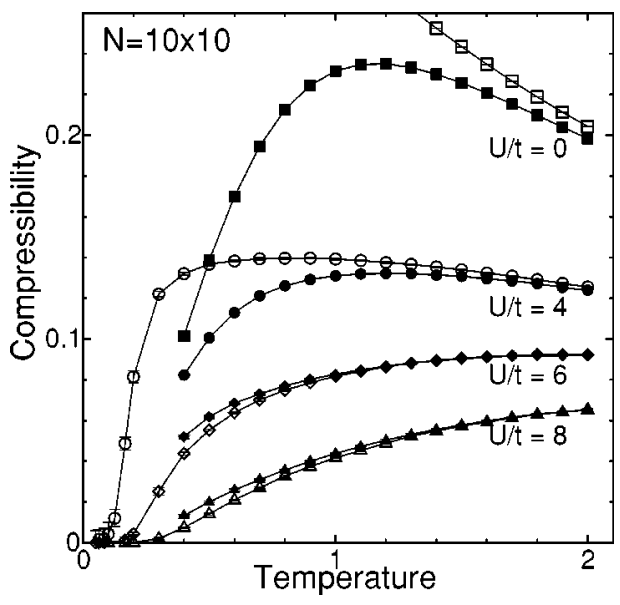

FIG. 1. Temperature dependence of the compressibility for a $N=10 \times 10$ lattice with interaction strengths $U / t=0$ (squares), 4 (circles), 6 (diamonds), and 8 (triangles). Solid symbols are for $\phi$ $=\pi$ and open symbols for $\phi=0$. When the interaction is small $(U / t \leqslant 4)$, the difference between $\phi=0$ and $\phi=\pi$ is large over wide range of temperatures.

If the system has an antiferromagnetic long-range order at zero temperature $S(\pi, \pi)$ shows diverging behavior as the temperature decreases.

\section{RESULTS}

First let us discuss effects of the interaction on the charge compressibility. We compare the result with those of the standard Hubbard model to clarify the effects of the flux. Figure 1 shows results of the charge compressibility $\kappa$. Due to the strong size dependence, data for $\phi=\pi$ at a very low temperature is omitted. When the interaction is small $(U / t$ $=4$ ), the difference in $\kappa$ between $\phi=0$ and $\phi=\pi$ is large over wide range of temperatures. In particular, at the lowest temprerature $(T / t=0.4)$, the value of the $\kappa$ is much closer to that of the noninteracting flux state $(\phi=\pi, U / t=0)$ than that of the Mott insulator $(\phi=0, U / t=4)$. It suggests that the effects of the flux is still relevant on the charge fluctuations even with the interaction. In addition, the $\kappa$ for $\phi=\pi$ and $U / t=4$ seems to vanish at $T=0$. Then this phase can be neither a standard Mott insulator nor a simple Fermi liquid. Of course we can not exclude all possibilities by the limited numerical results; our data suggests that the singular spectrum of the excitations which is characteristic in the flux state survives for weak interactions. On the other hand, when the interaction is strong $(U / t>8)$, the $\kappa$ shows the similar behavior independent of the flux. It implies that the local interaction $U$ dominates and the system is the Mott insulator for sufficiently strong coupling $(U \gg t)$. To determine the $U_{c}$ which divides the possible singular phase and the Mott insulator, one needs to see the temperature dependence of the $\kappa$ at very low temperature. If the system belongs to the flux phase, one expects the $\kappa$ to obey the power law, $\kappa / \operatorname{sim} T$, which reflects that the density of states $D /(\epsilon)$ near the Fermi energy is sinular. On the other hand, the $\kappa$ shows a thermally activated behavior for the Mott insulator. Within our present data, we estimate the $U_{c} / t$ is between 4 and 8 . 


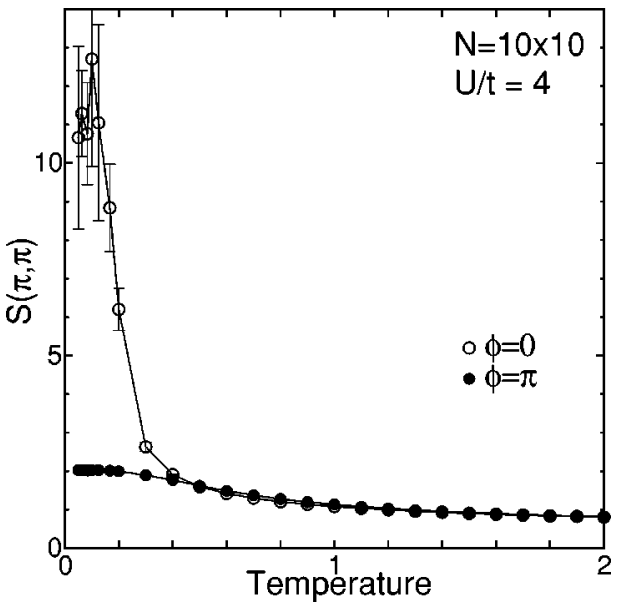

FIG. 2. The antiferromagnetic structure factor $S(\pi, \pi)$ as a function of temperature for $U / t=4$ on a $N=10 \times 10$ lattice. In the case of $\phi=0, S(\pi, \pi)$ diverges at low temperatures due to the formation of the antiferromagnetic order. On the other hand, for $\phi=\pi, S(\pi, \pi)$ does not show a diverging behavior.

Figure 2 shows the antiferromagnetic structure factor $S(\pi, \pi)$ versus temperatures. For the standard half-filled Hubbard model, since the ground state has an antiferromagnetic long-range order, $S(\pi, \pi)$ shows diverging behavior as the temperature decreases (it saturates when the antiferromagnetic correlation length is longer then the lattice size). On the other hand, for the flux phase, the formation of the long-range antiferromagnetic order is not observed for $U / t \leqslant 4$. According to the spin-wave theory, $S(\pi, \pi)$ at the zero temperature increases with lattice size as

$$
\frac{S(\pi, \pi)}{N}=\frac{m^{2}}{3}+O\left(N^{-1 / 2}\right),
$$

with $m$ the staggered magnetization which is an order parameter of an antiferromagnetic long-range order. Using this relation, we try to obtain $m^{2}$ by plotting $S(\pi, \pi) / N$ versus $N^{-1 / 2}=L^{-1}$. Figure 3 shows the plots of $S(\pi / \pi) / N$ for various sizes at $U / t=4$. The data are averaged over the temperatures where the system effectively reaches the zero temperature limit. For the standard Hubbard model, the data follow the relation (7) and the extrapolation value is finite indicating the existence of the antiferromagnetic long-range order. The value of $\mathrm{m}^{2}$ is consistent with the results of Refs. 17 and 18 . On the other hand, for the flux phase, the relation (7) with $m>0$ does not hold. It suggests absence of the antiferromagnetic long-range order at $T=0$, which is in contrast to the standard Hubbard model. When one discuss within the SDW mean-field theory, in the flux phase, the nesting instability is strongly suppressed due to the absence of the low energy excitations. The numerical results are consistent with this discussion at least in weak coupling.

If the interaction is sufficently strong $(U \gg t)$, the flux phase (4) is effectively described by the antiferromagnetic Heisenberg model, which isthe same as the standard Hubbard model. Therefore one can expect that the antiferromagnetic long-range order appears when the effects of the flux

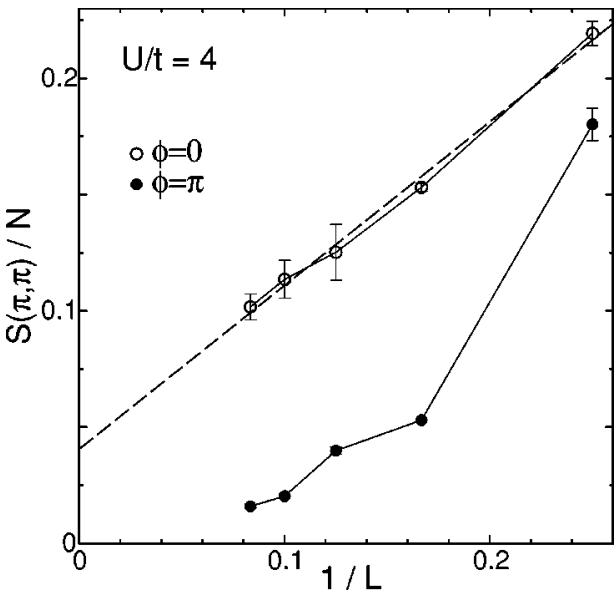

FIG. 3. The size dependence of the antiferromagnetic structure factor. The dashed line is a least-squares fit to the data for $\phi=0$. For $\phi=0$, the data extrapolate to a finite value, indicating that the ground state has an antiferromagnetic long-range order. On the other hand, for $\phi=\pi, S(\pi, \pi)$ versus $1 / L$ suggests the absent of the antiferromagnetic long-range order.

become irrelevant in the strong coupling $(U \gg t)$. Figure 4 shows the extrapolating plots $S(\pi, \pi) / N$ versus $L^{-1}$ for $U / t=4$ and $U / t=8$ in the presence of the flux. For the strong coupling $(U / t=8)$, the extrapolation value is finite within statistical errors. It suggests that the antiferromagnetic correlation enhances as $U / t$ increases and the long-range order appears at the finite value of the interaction strength $U / t \simeq 8$ although there is no long-range order at $U / t=4$. Then our data demonstrate the existence of the Mott transition at finite value of $U=U_{c}<8 t$. This estimate is consistent with that from the charge compressibility. This is clearly different from the standard Hubbard model, where the Mott transition occurs at an infinitesimally small interaction strength.

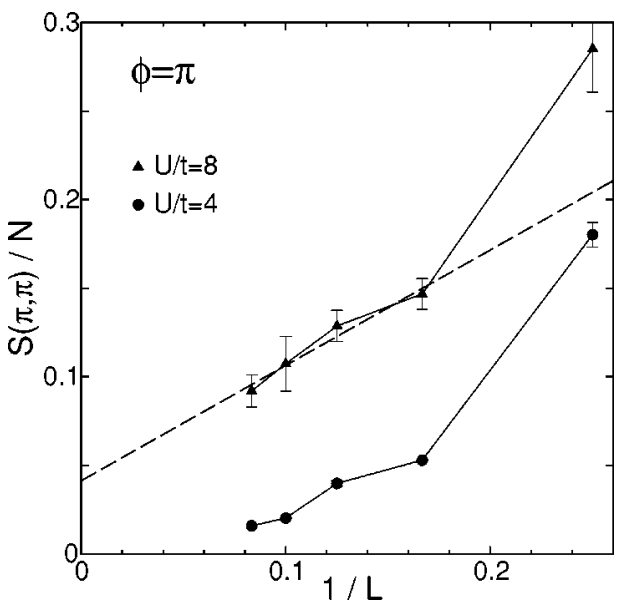

FIG. 4. The size dependence of the antiferromagnetic structure factor for $U / t=4$ (circles) and $U / t=8$ (triangles) in the presence of the flux. For $U / t=8$, the data extrapolates to a finite value indicating that the grounjd state has the antiferromagnetic long-range order. 


\section{DISCUSSION AND SUMMARY}

We have studied effects of the interaction in the flux phase. The Mott transition is focused using the quantum Monte Carlo method. Our results on the charge compressibility shows that effects of the flux is relevant for small $U / t$, while it becomes irrelevant when $U / t$ is sufficiently large. The antiferromagnetism, which is characteristic of the Mott insulator, is also strongly suppressed in the weak coupling region. This is due to the structure of the low energy excitations in the flux phase. It implies that the flux state with interaction leads to a new singular phase for $U<U_{c / 0}$. This phase is absent in the standard two-dimensional Hubbard model. Effects of the doping is also an interesting future issue in connection with the competition with the superconductivity.

\section{ACKNOWLEDGMENTS}

We are grateful to Y. Morita, M. Yamanaka, and Y. Kato for helpful comments. Y.H. is supported in part by Grant-inAid from the Ministry of Education, Science and Culture of Japan. The computation in this work has been done using the facilities of the Supercomputer Center, ISSP, University of Tokyo.
${ }^{1}$ I. Affleck and J. B. Marston, Phys. Rev. B 37, 3774 (1988).

${ }^{2}$ I. Affleck, Z. Zou, T. Hsu, and P. W. Anderson, Phys. Rev. B 38, 745 (1988).

${ }^{3}$ D. H. Kim and P. A. Lee, Ann. Phys. (N.Y.) 272, 130 (1999), and references therein.

${ }^{4}$ D.-H. Lee, Phys. Rev. B 60, 12429 (1999).

${ }^{5}$ D. A. Ivanov, P. A. Lee, and X.-G. Wen, Phys. Rev. Lett. 84, 3958 (2000).

${ }^{6}$ P. W. Leung, cond-mat/0007068 (unpublished).

${ }^{7}$ M. Yamanaka, W. Koshibae, and S. Maekawa, Phys. Rev. Lett. 81, 5604 (1998).

${ }^{8}$ M. Nakamura, J. Phys. Soc. Jpn. 68, 3123 (1999).

${ }^{9}$ S. Chakravarty, R. B. Laughlin, D. K. Morr, and C. Nayak, cond-mat/0005443 (unpublished).
${ }^{10}$ M. P. A. Fisher and E. Fradkin, Nucl. Phys. B251, 457 (1985).

${ }^{11}$ Y. Hatsugai and P. A. Lee, Phys. Rev. B 48, 4204 (1993).

${ }^{12}$ Y. Hatsugai, X.-G. Wen, and M. Kohmoto, Phys. Rev. B 56, 1061 (1997).

${ }^{13}$ Y. Morita and Y. Hatsugai, Phys. Rev. Lett. 79, 3728 (1997); Phys. Rev. B 58, 6680 (1998).

${ }^{14}$ Y. Hasegawa, P. Lederer, T. M. Rice, and P. B. Wiegmann, Phys. Rev. Lett. 63, 907 (1989).

${ }^{15}$ Y. Hasegawa, Y. Hatsugai, M. Kohmoto, and G. Montambaux, Phys. Rev. B 41, 9174 (1990).

${ }^{16}$ E. H. Lieb, Phys. Rev. Lett. 73, 2158 (1994).

${ }^{17}$ J. E. Hirsch, Phys. Rev. B 31, 4403 (1985).

${ }^{18}$ S. R. White, D. J. Scalapino, E. Y. Loh, J. E. Gubernatis, and R. T. Scalettar, Phys. Rev. B 40, 506 (1989). 